\title{
CONSTRAINTS ON OBSCURED STAR FORMATION IN HOST GALAXIES OF GAMMA-RAY BURSTS
}

\section{AUTHOR(S):}

Hatsukade, Bunyo; Hashimoto, Tetsuya; Ohta, Kouji; Nakanishi, Kouichiro; Tamura, Yoichi; Kohno, Kotaro

\section{CITATION:}

Hatsukade, Bunyo ...[et al]. CONSTRAINTS ON OBSCURED STAR FORMATION IN HOST GALAXIES OF GAMMA-RAY BURSTS. The Astrophysical Journal 2012, 748(2): 108.

\section{ISSUE DATE:}

2012-03-13

URL:

http://hdl.handle.net/2433/155086

\section{RIGHT:}

2012. The American Astronomical Society:; This is not the published version. Please cite only the published version.; この論文は出版社版で ありません。引用の際には出版社版をご確認ご利用ください。 
ACCEPted FOR PUBLiCATION in THE AstrophysicAl Journal

Preprint typeset using $\mathrm{LAT}_{\mathrm{E}} \mathrm{X}$ style emulateapj v. 8/13/10

\title{
CONSTRAINTS ON OBSCURED STAR FORMATION IN HOST GALAXIES OF GAMMA-RAY BURSTS
}

\author{
Bunyo Hatsukade ${ }^{1}$, Tetsuya Hashimoto ${ }^{2}$, Kouji Ohta ${ }^{1}$, Kouichiro Nakanishi ${ }^{2,3,4}$, Yoichi Tamura ${ }^{5}$, and \\ KOTARO KOHNO \\ Accepted for publication in the Astrophysical Journal
}

\begin{abstract}
We present the results of the 16-cm-waveband continuum observations of four host galaxies of gamma-ray bursts (GRBs) 990705, 021211, 041006, and 051022 using the Australia Telescope Compact Array. Radio emission was not detected in any of the host galaxies. The $2 \sigma$ upper limits on starformation rates derived from the radio observations of the host galaxies are $23,45,27$, and $26 M_{\odot} \mathrm{yr}^{-1}$, respectively, which are less than about 10 times those derived from UV/optical observations, suggesting that they have no significant dust-obscured star formation. GRBs 021211 and 051022 are known as the so-called "dark GRBs" and our results imply that dark GRBs do not always occur in galaxies enshrouded by dust. Because large dust extinction was not observed in the afterglow of GRB 021211, our result suggests the possibility that the cause of the dark GRB is the intrinsic faintness of the optical afterglow. On the other hand, by considering the high column density observed in the afterglow of GRB 051022, the likely cause of the dark GRB is the dust extinction in the line of sight of the GRB.

Subject headings: cosmology: observations — galaxies: high-redshift — galaxies: ISM — gamma-ray burst: individual (GRB 990705, GRB 021211, GRB 041006, GRB 051022) — radio continuum: galaxies
\end{abstract}

\section{INTRODUCTION}

One of the key issues in astronomy is to understand when and how stars were formed in the history of the universe. The cosmic star-formation history has been explored from local to high-redshift universe (e.g., Madau et al. 1996; Lilly et al. 1996). Long-duration gammaray bursts (GRBs) are thought to be powerful indicators of star-formation activity in the distant universe (e.g., Totani 1997) because (1) they are detectable even at $z>8$ (Tanvir et al. 2009; Salvaterra et al. 2009) and (2) they are considered to occur as a result of the deaths of massive stars (e.g., Stanek et al. 2003) and are closely associated with star formation in host galaxies. Host galaxies of GRBs have been studied mainly by UV, optical, and near-infrared (NIR) observations. The majority of GRB host galaxies are blue, subluminous, low-mass star-forming galaxies (e.g., Fynbo et al. 2003; Fruchter et al. 2006) with star-formation rates (SFRs) of $\sim 0.1$ $10 M_{\odot} \mathrm{yr}^{-1}$ (e.g., Savaglio et al. 2009; Svensson et al. 2010). On the other hand, massive star-forming host galaxies have also been discovered (e.g., Hashimoto et al. 2010; Svensson et al. 2011). Observations at the midIR, submillimeter, and radio ranges show that some GRB host galaxies have a large amount of star formation obscured by dust (e.g., Berger et al. 2003; Le Floc'h et al. 2006). It is found that star-formation activity at $z \gtrsim 1$ is

Electronic address: hatsukade@kusastro.kyoto-u.ac.jp

${ }^{1}$ Department of Astronomy, Kyoto University, Kyoto 606-8502

2 National Astronomical Observatory of Japan, 2-21-1 Osawa, Mitaka, Tokyo 181-8588

3 Joint ALMA Office, Alonso de Córdova 3107, Vitacura, Santiago 763 0355, Chile

4 The Graduate University for Advanced Studies (Sokendai), 2-

21-1 Osawa, Mitaka, Tokyo 181-8588, Japan

${ }^{5}$ Institute of Astronomy, the University of Tokyo, 2-21-1 Osawa, Mitaka, Tokyo 181-0015

${ }^{6}$ Research Center for the Early Universe, University of Tokyo, 7-3-1 Hongo, Bunkyo, Tokyo 113-0033, Japan dominated by IR-luminous dusty galaxies (e.g., Le Floc'h et al. 2005; Takeuchi et al. 2005). If GRBs trace starforming activity, they could occur in dust-obscured regions (e.g., Djorgovski et al. 2001).

Dust extinction is a possible cause of mysterious events of so-called "dark GRBs" (e.g., Fynbo et al. 2001; Perley et al. 2009). Dark GRBs, which are defined by the faintness of the optical afterglow compared with that expected from X-ray afterglow (Jakobsson et al. 2004), make up about 25-40\% of GRBs (Fynbo et al. 2009; Greiner et al. 2011). The possible explanations for this are that (1) optical afterglows are obscured by dust within host galaxies distributed globally or in the vicinity of GRBs; (2) optical afterglow is intrinsically faint owing to low-luminous GRB events, fast-declining afterglows, or the low-density of the ambient medium; and (3) GRBs are at high redshift and the optical afterglows are absorbed by intervening neutral hydrogen. Detailed studies on GRB host galaxies have been mostly conducted by using UV/optical observations, which are biased toward less dusty galaxies. Recent extensive followup campaigns of GRBs and their host galaxies suggest that a large fraction of GRBs occur in dusty environments (e.g., Perley et al. 2009; Greiner et al. 2011). In order to obtain a clear understanding of GRB host galaxies, it is essential to study them using methods that are unaffected by dust extinction. Radio observations are effective measures for examining star-forming activity without the effect of dust obscuration. So far, only a small fraction of GRB host galaxies have been observed at radio wavelength (e.g., Berger et al. 2003; Stanway et al. 2010).

In this paper, we report $16-\mathrm{cm}(2-\mathrm{GHz})$ waveband continuum observations for four GRB host galaxies. These results were obtained by using the Australia Telescope Compact Array (ATCA). Section 2 describes the target GRB host galaxies. Section 3 outlines the observations, 
data reduction, and results. In Section 4, the constraints on SFRs are derived and obscured star formation in the GRB host galaxies is discussed as well as the cause of dark GRBs. In Section 5, a summary of the findings is presented. Throughout the paper, we adopt a cosmology with $H_{0}=70 \mathrm{~km} \mathrm{~s}^{-1} \mathrm{Mpc}^{-1}, \Omega_{\mathrm{M}}=0.3$, and $\Omega_{\Lambda}=0.7$.

\section{TARGETS}

We observed the host galaxies of GRBs 990705, 021211, 041006, and 051022. In order to obtain tight constraints on the obscured star formation, we selected the targets at $z \leq 1$ with SFRs well derived from UV/optical observations. The UV/optical SFRs, as hereafter stated, are corrected for dust extinction.

The GRB 990705 host galaxy is a star-forming galaxy at $z=0.8424$ (Le Floc'h et al. 2002). The SFR derived from UV luminosity is SFR(UV) 5-8 (Le Floc'h et al. 2002) and $3.31 M_{\odot} \mathrm{yr}^{-1}$ (Svensson et al. 2010). The SFR based on the $[\mathrm{OII}]$ line is $\mathrm{SFR}([\mathrm{OII}])=6.96 M_{\odot} \mathrm{yr}^{-1}$ (Savaglio et al. 2009). The galaxy is detected with Spitzer at 4.5 and $24 \mu \mathrm{m}$ (Le Floc'h et al. 2006). The IR luminosity inferred from the $24-\mu \mathrm{m}$ detection is $1.8_{-0.6}^{+2.1} \times 10^{11} L_{\odot}$, classifying the galaxy as a luminous IR galaxy (LIRG). The SFR derived from the IR luminosity is $\mathrm{SFR}(\mathrm{IR})=32_{-11}^{+37} M_{\odot} \mathrm{yr}^{-1}$. Castro Cerón et al. (2006) derived a SFR of $4.5-173 M_{\odot} \mathrm{yr}^{-1}$ based on the opticalradio spectral energy distribution (SED) that included the Spitzer photometry. The SFR derived from the IR observations is higher than the extinction-corrected SFR derived from UV/optical observations shown above, suggesting the existence of dust-obscured star formation, which cannot be traced at UV/optical wavelengths. Berger et al. (2003) failed to detect significant radio continuum emission at $1.39 \mathrm{GHz}\left(S_{1.39 \mathrm{GHz}}=52 \pm 32 \mu \mathrm{Jy}\right.$, which corresponds to a $\left.\mathrm{SFR}=190 \pm 165 M_{\odot} \mathrm{yr}^{-1}\right)$.

GRB 021211 at $z=1.006$ (Vreeswijk et al. 2003) is classified as a dark GRB owing to its faintness of optical afterglow (Fox et al. 2003; Li et al. 2003; Crew et al. 2003). Della Valle et al. (2003) found supernova signatures in the spectrum of the optical afterglow. The $\mathrm{UV}$-derived SFR is $\mathrm{SFR}(\mathrm{UV})=3.01 M_{\odot} \mathrm{yr}^{-1}$ (Savaglio et al. 2009) and $6.95 M_{\odot} \mathrm{yr}^{-1}$ (Svensson et al. 2010).

The GRB 041006 associated with a supernova occurred at $z=0.716$ (Fugazza et al. 2004; Stanek et al. 2005; Soderberg et al. 2006). The SFRs of the host galaxy is $\operatorname{SFR}\left(\left[\mathrm{O}_{\mathrm{II}}\right]\right)=0.34 M_{\odot} \mathrm{yr}^{-1}$ (Savaglio et al. 2009) and $\mathrm{SFR}(\mathrm{UV})=1.17 M_{\odot} \mathrm{yr}^{-1}$ (Svensson et al. 2010).

GRB 051022 is another dark GRB whose optical/NIR afterglow was undetected. Millimeter and radio interferometer observations detected the afterglow and pinpointed the position of the host galaxy at $z=0.807$ (Castro-Tirado et al. 2007; Rol et al. 2007). The host galaxy is a luminous blue compact star-forming galaxy with a rest-frame $B$-band magnitude of $M_{B}=$ -21.8 mag (Castro-Tirado et al. 2007). The optical/NIR afterglow was not detected despite a bright X-ray afterglow and a high absorption column density. The X-ray spectrum of the afterglow shows a strong absorption corresponding to $N_{\mathrm{H}}=3-9 \times 10^{22} \mathrm{~cm}^{-2}$ (Nakagawa et al. 2006; Castro-Tirado et al. 2007). The galaxy shows active star formation with $\operatorname{SFR}\left(\left[\mathrm{O}_{\mathrm{II}}\right]\right)=34.64 M_{\odot} \mathrm{yr}^{-1}$ (Savaglio et al. 2009) and $\operatorname{SFR}(\mathrm{UV})=23.85 M_{\odot} \mathrm{yr}^{-1}$
TABLE 1

Results of the ATCA 16 CM OBservations

\begin{tabular}{cccc}
\hline GRB & $z$ & $\begin{array}{c}\text { rms noise } \\
\left(\mu \mathrm{Jy} \mathrm{beam}^{-1}\right)\end{array}$ & $\begin{array}{c}\text { SFR } \\
\left(M_{\odot} \mathrm{yr}^{-1}\right)\end{array}$ \\
\hline 990705 & 0.842 & 8.65 & $<23$ \\
021211 & 1.006 & 11.4 & $<45$ \\
041006 & 0.712 & 15.0 & $<27$ \\
051022 & 0.807 & 11.0 & $<26$ \\
\hline
\end{tabular}

Note. - Upper limits are $2 \sigma$.

(Svensson et al. 2010).

\section{OBSERVATIONS AND RESULTS}

The ATCA observations were conducted on September 14 and 15, 2011 using 6 antennas in the 6B array configuration (baseline lengths of 214-5969 m). The Compact Array Broadband Backend (CABB) was used in the CFB 1M-0.5k mode with $2048 \times 1-\mathrm{MHz}$ channels centered at $2100 \mathrm{MHz}$ (16-cm band). PKS 1934-638 was observed at the beginning of the observations as a flux and bandpass calibrator. PKS 0252-712, PKS 0741-063, PKS 0106+013, and PKS 0019-000 were observed as phase calibrators for the host galaxies of GRBs 990705, 021211, 041006, and 051022, respectively. To fill in the $u v$ plane as much as possible, we performed cyclic observations of the targets with a short integration time (15-30 minutes).

Data reduction and imaging were carried out using the MIRIAD package (Sault et al. 1995). The $16-\mathrm{cm}$ band is severely affected by radio frequency interference, and hence, we carefully flagged the data. Because the $\mathrm{CABB}$ data cover a large fractional bandwidth at the 16 -cm band, the calibrations were performed by using a multi-frequency strategy to account for the amplitude dependence on frequency. The calibrated data were imaged with using multi-frequency synthesis. Uniform weighting was adopted to minimise the sidelobe levels. The image was deconvolved with the task MFCLEAN.

We did not detect radio emission from any of the host galaxies. The rms noise levels are 8.7, 11, 15, $11 \mu \mathrm{Jy} \mathrm{beam}^{-1}$ for GRBs 90705, 021211, 041006, and 051022, respectively, which are measured at an area near the central position without any source or significant sidelobes. In the following section, we discuss the constraints using the $2 \sigma$ upper limits.

\section{DISCUSSION}

\subsection{Constraints on Star-Formation Rate}

We derive the $2 \sigma$ upper limits on the SFRs of the host galaxies using the rms noise levels. We use the relation between radio flux and the SFR for starburst galaxies derived by Yun \& Carilli (2002) as follows:

$$
\begin{aligned}
S\left(\nu_{\mathrm{obs}}\right)= & \left\{25 f_{\mathrm{nth}} \nu_{0}^{-\alpha}+0.71 \nu_{0}^{-0.1}\right. \\
& \left.+1.3 \times 10^{-6} \frac{\nu_{0}^{3}\left[1-e^{-\left(\nu_{0} / 2000\right)^{\beta}}\right]}{e^{0.048 \nu_{0} / T_{d}}-1}\right\} \\
& \times \frac{(1+z) \mathrm{SFR}}{D_{L}^{2}},
\end{aligned}
$$

where $S\left(\nu_{\mathrm{obs}}\right)$ is the observed flux density in Jy, $\nu_{0}$ is the rest frequency in $\mathrm{GHz}, f_{\mathrm{nth}}$ is a scaling factor, $\alpha$ is the 
synchrotron spectral index, $\beta$ is the dust emissivity, $T_{d}$ is the dust temperature in $\mathrm{K}, D_{L}$ is the luminosity distance in $\mathrm{Mpc}$, and $\mathrm{SFR}$ is in $M_{\odot} \mathrm{yr}^{-1}$. The 1st, 2nd, and 3rd term on the right-hand side represent the contribution of non-thermal synchrotron emission, free-free emission, and thermal dust emission, respectively. We adopt $\alpha=0.6, \beta=1.35, T_{d}=58 \mathrm{~K}$, and $f_{\text {nth }}=1$, which are selected for IR-selected starburst galaxies (Yun \& Carilli 2002) and were used in previous radio studies on GRB host galaxies (Berger et al. 2003; Stanway et al. 2010). The 1st non-thermal term dominates the flux density at the observed frequency of $2.1 \mathrm{GHz}$. The $2 \sigma$ upper limits on SFRs derived using the equation (1) are 23, 45, 27, and $26 M_{\odot} \mathrm{yr}^{-1}$ for the host galaxies of GRBs 990705, 021211, 041006, and 051022, respectively (Table 1). The upper limits on SFRs are less than about 10 times the UV/optical SFRs (Section 2), suggesting no significant dust-obscured star formation, which is undetectable at UV/optical wavelengths. Although the Spitzer observations indicate that the GRB 990705 host galaxy has obscured star-forming activity (Le Floc'h et al. 2006), our $2 \sigma$ upper limit on SFR is consistent with the SFRs derived from the IR observations after considering their uncertainties.

In Figure 1, we compare the SFRs of the GRB host galaxies derived from the radio observations with those derived from the UV/optical observations. The data are taken from our results and literature. To check whether there is a difference in obscured star formation between dark and non-dark GRB host galaxies, we differentiate the dark GRBs (solid symbols) from the non-dark GRBs (open symbols). The plot shows, that in most cases, the GRB host galaxies do not have a large amount of obscured star formation irrespective of whether they are dark or not. This indicates that the dark GRB host galaxies are not always dusty star-forming galaxies (e.g., Le Floc'h et al. 2006). Recent deep, systematic follow-up observations of GRBs at the optical/NIR region suggest that a significant fraction of GRBs take place in dustobscured regions (e.g., Perley et al. 2009; Greiner et al. 2011). It is possible that the sample selection could affect the properties of the GRB host galaxies. We collect the host galaxies studied with UV/optical wavelengths, which are biased against dusty galaxies. The sample size studied here is still small and more observations with extinction-free methods are needed. Another possible reason is that the GRB host galaxies studied here are all at $z \lesssim 1$, where low-luminosity galaxies account for the large proportion of star-forming activity (Le Floc'h et al. 2005). Recent IR observations uncovered that star formation at $z \gtrsim 1$ is dominated by IR-luminous dusty galaxies (e.g., Le Floc'h et al. 2005; Takeuchi et al. 2005) and the fraction of dusty GRB host galaxies might increase at higher-redshift.

\subsection{Cause of Dark GRBs}

There is a debate regarding the cause of dark GRBs. The possible explanations are that they are (1) obscured by dust in host galaxies, (2) intrinsically faint, and (3) at high redshifts. We do not need to consider the possibility of high-redshift GRBs for our sample because they are at $z \leq 1$. Given the null results of our deep radio observations, it is not likely that the dark GRB host galaxies in our sample are globally obscured by dust.

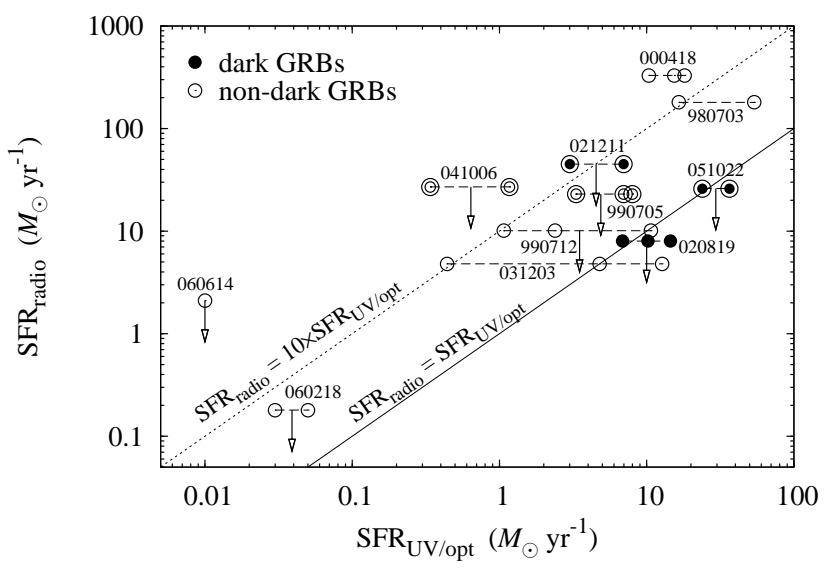

FIG. 1.- Comparison between SFRs derived from radio observations and extinction-corrected SFRs derived from UV/optical observations. We plot the results of this work and previous works: Radio data are obtained from Berger et al. (2003) and Stanway et al. (2010) and UV/optical data are obtained from Gorosabel et al. (2003); Savaglio et al. (2009); Svensson et al. (2010); Levesque et al. (2010a,b). Host galaxies of this work are encircled. Filled and open symbols denote dark and non-dark GRBs, respectively. Diagonal solid and dotted lines indicate the radio SFRs equal to and 10 times the UV/optical SFRs, respectively. Horizontal dashed lines represent the GRB host galaxies with different UV/optical SFRs in literature.

The optical afterglow spectrum of GRB 021211 shows that the extinction in the line of sight of the GRB is negligible (Holland et al. 2004). The observed optical afterglow of GRB 021211 is thought to be intrinsically faint owing to the fast fading afterglow (e.g., Fox et al. 2003; Crew et al. 2003; Li et al. 2003); our results support this scenario.

Our results suggest that the host galaxy of GRB 051022 is not likely a dusty star-forming galaxy. The X-ray afterglow spectrum suggests a high column density of neutral hydrogen (Nakagawa et al. 2006; Castro-Tirado et al. 2007). Castro-Tirado et al. (2007) state that the extinction in the host galaxy of GRB $051022\left(A_{V}=1.0\right)$ derived from its SED cannot account for the afterglow properties, assuming a Galactic dust-to-gas ratio. A possible explanation for the cause of dark GRB 051022 is that the burst occurred inside a giant molecular cloud, where a high column density is expected (Castro-Tirado et al. 2007; Rol et al. 2007). In some GRB afterglows, the absorption features of $\mathrm{H}_{2}$ and $\mathrm{CO}$ molecules along the line of sight of GRBs have actually been observed at the same redshifts of the GRBs (Fynbo et al. 2006a; Prochaska et al. 2009; Sheffer et al. 2009). Measuring the amount of molecular gas in the GRB host galaxies is important, but, so far, CO emission has not been detected (Kohno et al. 2005; Endo et al. 2007; Hatsukade et al. 2007, 2011). Future deep and high-resolution observations of molecular gas will be required to understand the environments of GRB origins.

\section{SUMMARY}

We performed 16-cm continuum observations of the host galaxies of GRBs 990705, 021211, 041006, and 051022 using the ATCA. GRBs 021211 and 051022 are known as dark GRBs. We failed to detect radio emission in any of the host galaxies. The $2 \sigma$ upper limits on the SFRs of the host galaxies of GRBs 990705, 021211, 
041006, and 051022 derived from the radio observations are $23,45,27$, and $26 M_{\odot} \mathrm{yr}^{-1}$, respectively. The upper limits on SFRs are less than about 10 times those derived from the UV/optical observations, suggesting that they have no significant dust-obscured star formation, which cannot be traced at UV/optical wavelengths. This indicates that dark GRBs do not necessarily occur in galaxies enshrouded by dust. The afterglow spectrum of GRB 021211 shows that the dust extinction in the line of sight to the GRB is negligible, and our results suggests the possibility that GRB 021211 is intrinsically faint. Given the high column density observed in the afterglow spectrum of GRB 051022, the likely explanation for the dark GRB is dust extinction in the line of sight of the GRB.
We would like to acknowledge Sarah Maddison, Robin Wark, Yiannis Gonidakis, and the ATCA staffs for the help they provided during the observation. We thank the referee for helpful comments and suggestions. The Australia Telescope Compact Array is part of the Australia Telescope National Facility which is funded by the Commonwealth of Australia for operation as a National Facility managed by CSIRO. BH is supported by Research Fellowship for Young Scientists from the Japan Society of the Promotion of Science (JSPS). TH and KO are supported by the grant-in-aid for Scientific Research on Priority Area (19047003) from the Ministry of Education, Culture, Sports, Science and Technology (MEXT) of Japan.

\section{REFERENCES}

Berger, E., Cowie, L. L., Kulkarni, S. R., Frail, D. A., Aussel, H., \& Barger, A. J. 2003, ApJ, 588, 99

Castro Cerón, J. M., Michałowski, M. J., Hjorth, J., Watson, D. Fynbo, J. P. U., \& Gorosabel, J. 2006, ApJ, 653, L85

Castro-Tirado, A. J., Bremer, M., McBreen, S., et al. 2007, A\&A, 475, 101

Crew, G. B., Lamb, D. Q., Ricker, G. R., et al. 2003, ApJ, 599, 387

Della Valle, M., Malesani, D., Benetti, S., et al. 2003, A\&A, 406, L33

Djorgovski, S. G., Frail, D. A., Kulkarni, S. R., Bloom, J. S.,

Odewahn, S. C., \& Diercks, A. 2001, ApJ, 562, 654

Endo, A., et al. 2007, ApJ, 659, 1431

Fox, D. W., Price, P. A., Soderberg, A. M., et al. 2003, ApJ, 586, L5

Fruchter, A. S., et al. 2006, Nature, 441, 463

Fugazza, D., Fiore, F., Covino, S., et al. 2004, GRB Coordinates Network, 2782, 1

Fynbo, J. U., et al. 2001, A\&A, 369, 373

Fynbo, J. P. U., et al. 2003, A\&A, 406, L63

Fynbo, J. P. U., et al. 2009, ApJS, 185, 526

Fynbo, J. P. U., Starling, R. L. C., Ledoux, C., et al. 2006a, A\&A, 451, L47

Fynbo, J. P. U., Watson, D., Thöne, C. C., et al. 2006b, Nature, 444,1047

Gorosabel, J., et al. 2003, A\&A, 409, 123

Greiner, J., et al. 2011, A\&A, 526, A30

Hashimoto, T., Ohta, K., Aoki, K., et al. 2010, ApJ, 719, 378

Hatsukade, B., et al. 2007, PASJ, 59, 67

Hatsukade, B., Kohno, K., Endo, A., Nakanishi, K., \& Ohta, K. 2011, ApJ, 738, 33

Holland, S. T., Bersier, D., Bloom, J. S., et al. 2004, AJ, 128, 1955

Jakobsson, P., Hjorth, J., Fynbo, J. P. U., Watson, D., Pedersen,

K., Björnsson, G., \& Gorosabel, J. 2004, ApJ, 617, L21

Kohno, K., et al. 2005, PASJ, 57, 147

Le Floc'h, E., Duc, P.-A., Mirabel, I. F., et al. 2002, ApJ, 581, L81

Le Floc'h, E., Charmandaris, V., Forrest, W. J., Mirabel, I. F., Armus, L., \& Devost, D. 2006, ApJ, 642, 636

Le Floc'h, E., Papovich, C., Dole, H., et al. 2005, ApJ, 632, 169

Levesque, E. M., Berger, E., Kewley, L. J., \& Bagley, M. M. 2010a, AJ, 139, 694
Levesque, E. M., Kewley, L. J., Graham, J. F., \& Fruchter, A. S. 2010b, ApJ, 712, L26

Li, W., Filippenko, A. V., Chornock, R., \& Jha, S. 2003, ApJ, 586, L9

Lilly, S. J., Le Fevre, O., Hammer, F., \& Crampton, D. 1996, ApJ, 460, L1

Madau, P., Ferguson, H. C., Dickinson, M. E., et al. 1996 , MNRAS, 283, 1388

Nakagawa, Y. E., Yoshida, A., Sugita, S., et al. 2006, PASJ, 58 L35

Perley, D. A., et al. 2009, AJ, 138, 1690

Prochaska, J. X., Sheffer, Y., Perley, D. A., et al. 2009, ApJ, 691, L27

Rol, E., van der Horst, A., Wiersema, K., et al. 2007, ApJ, 669, 1098

Salvaterra, R., et al. 2009, Nature, 461, 1258

Savaglio, S., Glazebrook, K., \& Le Borgne, D. 2009, ApJ, 691, 182

Sault, R. J., Teuben, P. J., \& Wright, M. C. H. 1995, Astronomical Data Analysis Software and Systems IV, 77, 433

Sheffer, Y., Prochaska, J. X., Draine, B. T., Perley, D. A., \& Bloom, J. S. 2009, ApJ, 701, L63

Soderberg, A. M., Kulkarni, S. R., Price, P. A., et al. 2006, ApJ, 636, 391

Stanek, K. Z., et al. 2003, ApJ, 591, L17

Stanek, K. Z., Garnavich, P. M., Nutzman, P. A., et al. 2005, ApJ, 626, L5

Stanway, E. R., Davies, L. J. M., \& Levan, A. J. 2010, MNRAS, 409, L74

Svensson, K. M., Levan, A. J., Tanvir, N. R., Fruchter, A. S., \& Strolger, L.-G. 2010, MNRAS, 405, 57

Svensson, K. M., Tanvir, N. R., Perley, D. A., et al. 2011, arXiv:1109.3167

Takeuchi, T. T., Buat, V., \& Burgarella, D. 2005, A\&A, 440, L17

Tanvir, N. R., et al. 2009, Nature, 461, 1254

Totani, T. 1997, ApJ, 486, L71

Vreeswijk, P., Fruchter, A., Hjorth, J., \& Kouveliotou, C. 2003, GRB Coordinates Network, 1785, 1

Yun, M. S., \& Carilli, C. L. 2002, ApJ, 568, 88 